\title{
Neue Referenzsonden auf der Basis von Wasserstoffelektroden für die Lebensmittelanalytik
}

\author{
Johannes Schwarz $^{1)}$, Alexander Hörig ${ }^{1)}$, Wolfram Oelßner ${ }^{1)}$, Winfried Vonau ${ }^{1)}$, \\ Hans-Joachim Kohnke ${ }^{2)}$ \\ ${ }^{1)}$ Kurt-Schwabe-Institut für Mess- und Sensortechnik e. V. Meinsberg \\ ${ }^{2)}$ Gaskatel Gesellschaft für Gassysteme durch Katalyse und Elektrochemie mbH
}

\section{Zusammenfassung}

Zur pH-Bestimmung in Lebensmittelproben wurden neue, auf Wasserstoffelektroden basierende Referenzsonden eingesetzt. Diese Elektroden zeichnen sich durch einfachen Elektrodenaufbau mit einer internen Wasserstoffquelle, hohe mechanische Stabilität, leichte Handhabbarkeit, robuste Bauweise und stabile Potentialwerte aus und können als glasfreie Alternative zu konventionellen $\mathrm{Ag} / \mathrm{AgCl}, \mathrm{Cl}^{-}$Elektroden eingesetzt werden. In dem vorliegenden Beitrag werden der konstruktive Aufbau der Elektroden beschrieben und Ergebnisse von potentiometrischen $\mathrm{pH}$-Bestimmungen in Milchprodukten sowie von Untersuchungen zur zeitlichen Alterung der Lebensmittelproben vorgestellt, die unter Verwendung der neuen Referenzsonden durchgeführt worden sind.

\section{Einleitung}

Die Standard-Wasserstoffelektrode (SHE, standard hydrogen electrode) besitzt in der Elektrochemie eine zentrale Bedeutung als Referenzsystem und Nullpunkt der elektrochemischen Potentialskala. Sie besteht im Wesentlichen aus einem platinierten Platinblech, das in eine HCl-Lösung der Protonenaktivität $1 \mathrm{~mol} / \mathrm{L}$ eintaucht und von Wasserstoffgas unter einem Druck von 101,3 kPa umspült wird. Das Wasserstoffgas wird teilweise an der Platinelektrode adsorbiert und bildet gemäß Gleichung

$$
\mathrm{H}_{2}+2 \mathrm{H}_{2} \mathrm{O} \rightleftarrows 2 \mathrm{H}_{3} \mathrm{O}^{+}+2 \mathrm{e}^{-}
$$

ein Gleichgewicht mit den sich in Lösung befindlichen hydratisierten Wasserstoffionen. Definitionsgemäß ist das Potential des Elektronendurchtritts an der Platinelektrode unter den genannten Standardbedingungen bei allen Temperaturen gleich Null.

Auf Grund des hohen apparativen Aufwands und der schwierigen Handhabung sind diese Elektroden in ihrer ursprünglichen Ausführungsform für einen routinemäßen und mobilen Einsatz nicht geeignet und werden heute in der praktischen Messtechnik kaum noch angewendet [1-3]. Sie werden nur noch vereinzelt für besondere Messaufgaben, beispielsweise die Überprüfung von pH-Pufferlösungen und für Kontrolluntersuchungen von definierten Messlösungen, herangezogen [4].

Erst im Jahre 1985 konnten mit der Erfindung einer kleinen, gut regelbaren galvanischen Zelle zur Entwicklung von Wasserstoff die oben genannten Nachteile behoben, der konstruktive Aufbau der Wasserstoffelektrode wesentlich vereinfacht und damit das Interesse daran erneut geweckt werden [5]. Auf Basis dieser Entwicklung wurden von A. Winsel in mehreren Patenten [6-8] stabförmige Wasserstoffelektroden mit internen, in den Kunststoff-Elektrodenkörper integrierten Wasserstoffquellen vorgeschlagen, die den Praxiseinsatz wesentlich erleichtern. Von der Fa. Gaskatel Gesellschaft für Gassysteme durch Katalyse und Elektrochemie $\mathrm{mbH}$ werden derartige Wasserstoffelektroden mit integrierten Wasserstoffquellen hergestellt und unter dem Namen Hydroflex ${ }^{\circledR}$ kommerziell angeboten. Durch die nachfolgend beschriebene Modifizierung mit definierten Elektrolyten können die Elektroden auch als potentialstabile Referenzsonden in realen Proben eingesetzt werden. 


\section{Elektrodenaufbau}

In Bild 1 sind die äußere Gestaltung und die wesentlichen Komponenten der neuen Referenzsonden auf Basis der Hydroflex ${ }^{\circledR}$-Wasserstoffelektrode angegeben. Der Elektrodenkörper besteht aus einem in den für die in Betracht gezogenen Anwendungen chemisch beständigen Werkstoff. In den Elektrodenkopf ist die regelbare Wasserstoffquelle integriert. Der aus dieser kontinuierlich ausströmende in-situ erzeugte Wasserstoff gelangt durch ein in dem Elektrodenkörper vorhandenes Rohr zu der aus Palladium, Platin und Polytetrafluorethylen (PTFE) bestehenden Gasdiffusionselektrode. Diese taucht in einen Elektrolytbecher ein, der mit einem säure- bzw. salzhaltigen Elektrolyten definierter Zusammensetzung gefüllt ist, und liefert ein durch dessen jeweilige Wasserstoffionenaktivität festgelegtes konstantes Referenzpotential. Die erforderliche elektrolytische Verbindung zwischen dem Elektrolytreservoir und dem Messmedium erfolgt über eine mikroporöse Membran, die sich durch geringe Ausflussrate und große Beständigkeit auszeichnet, was im Hinblick auf die Langzeitstabilität der Referenzelektroden wesentlich ist.

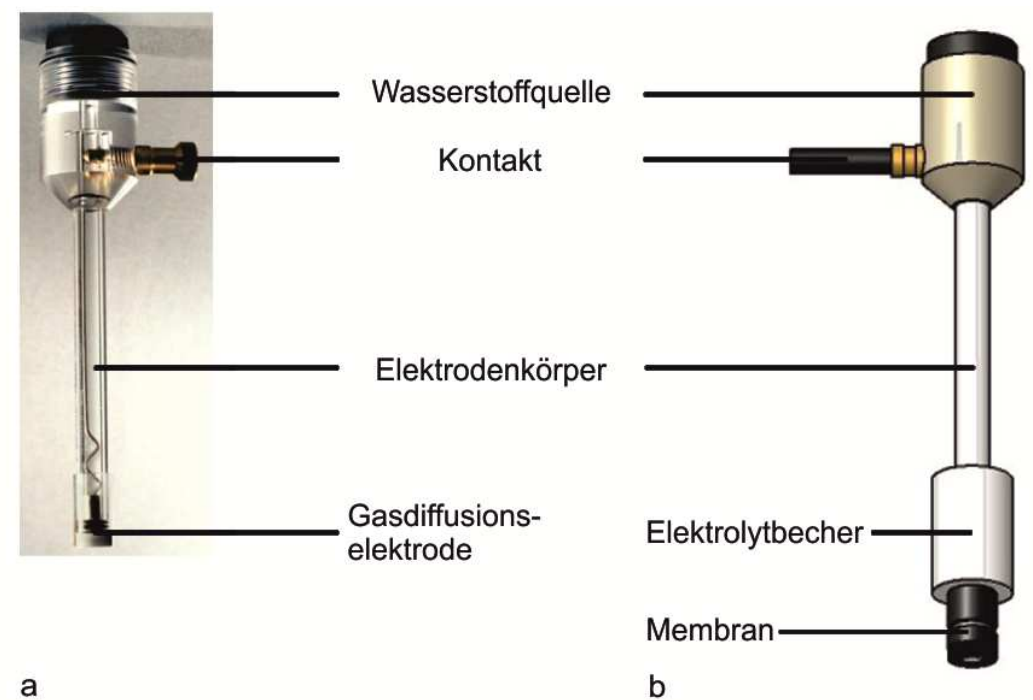

Bild 1 a) Hydroflex ${ }^{\circledR}$-Wasserstoffelektrode; b) Neue Referenzsonde

\section{Messergebnisse}

Die neuen Elektroden wurden in verschiedenen pH-Puffersystemen als Mess- und Referenzelektroden erprobt und gegen die Standard-Wasserstoffelektrode kalibriert. Bild 2 zeigt das potentiometrische Ansprechverhalten unter Verwendung der neuen Referenzsonden bei Messungen in $\mathrm{pH}$ Pufferlösungen und verschiedenen Lebensmittelproben, wobei als Messelektroden konventionelle $\mathrm{pH}-$

Glaselektroden verwendet wurden.

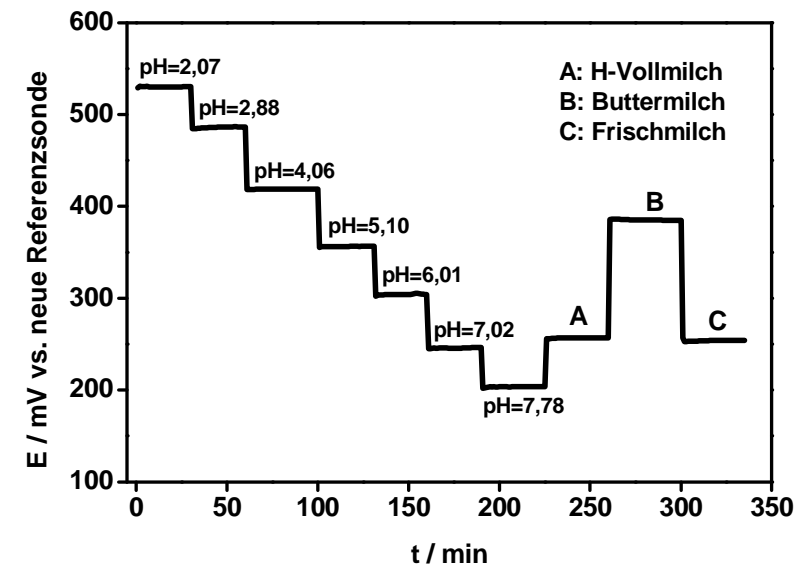

a

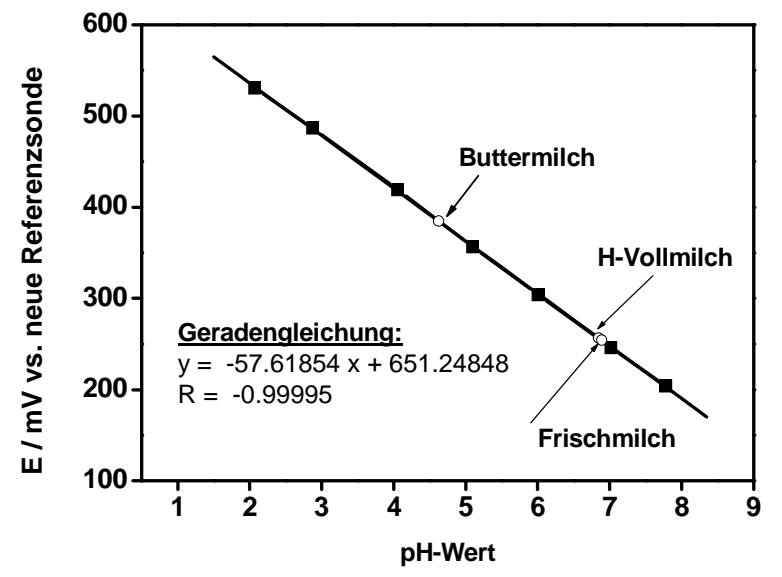

b

Bild 2 a) Potentiometrisches Ansprechverhalten in Pufferlösungen und Lebensmittelproben, b) Kalibrierkurve einer $\mathrm{pH}$-Glaselektrode bei Verwendung der neuen Referenzsonde auf Basis der Wasserstoffelektrode 
Für die Kalibrierung der Sensoren wurden als pH-Puffersysteme NBS-Puffer (pH-Werte: 1,68, 4,01, 6,86 und 9,18) und auch Britton-Robinson-Puffer mit konstanter Ionenstärke ( $\mathrm{pH}$-Werte von 2 bis 9) verwendet. Die ermittelte $\mathrm{pH}$-Sensitivität erreichte im untersuchten $\mathrm{pH}$-Bereich näherungsweise den sich aus der Nernst'schen Gleichung ergebenden Wert.

In Tafel 1 sind die ermittelten $\mathrm{pH}-$ Werte in verschiedenen Milchprodukten zusammengefasst. Die mit den neuen Referenzsonden erzielten $\mathrm{pH}$-Werte stimmen mit den mit konventionellen $\mathrm{pH}$ Einstabmessketten erzielten Werten und Literaturangaben überein.

Tafel $1 \mathrm{pH}-$ Werte verschiedener Milchprodukte

\begin{tabular}{|c|c|c|}
\hline Milchprodukte & $\begin{array}{c}\text { pH-Glaselektrode } \\
\text { vs. neue Referenzsonde }\end{array}$ & $\begin{array}{c}\text { kommerzielle } \\
\text { pH-Einstabmesskette }\end{array}$ \\
\hline H-Vollmilch (3,5\% Fett) & 6,84 & 6,70 \\
\hline Frischmilch (3,5\% Fett) & 6,89 & 6,77 \\
\hline Buttermilch & 4,27 & 4,10 \\
\hline Kefir & 4,87 & 4,61 \\
\hline Sauermilch & 3,54 & 3,57 \\
\hline
\end{tabular}

Die Änderung des pH-Wertes bei der zeitlichen Alterung von verschiedenen Milchproben ist in Bild 3 am Beispiel einer Frischmilch- und einer H-Milch-Probe dargestellt. Als Messelektroden wurden konventionelle $\mathrm{pH}$-Glaselektroden, als Referenzelektroden die beschriebenen Wasserstoffelektroden eingesetzt. Zum Vergleich sind Kurvenverläufe, die mit kommerziellen pH-Einstabmessketten (gestrichelte Linien) erhalten wurden, dargestellt. Die Alterung der Milchproben ist erkennbar durch Abnahme des $\mathrm{pH}$-Wertes. Bei der Frischmilchprobe beginnt die Alterung nach ca. 16 Tagen, während bei der H-Vollmilchprobe erst nach ca. 28 Tagen eine deutliche Abnahme des pH-Wertes zu beobachten ist. Die Milchproben wurden während dieser Zeit bei Raumtemperatur gelagert.
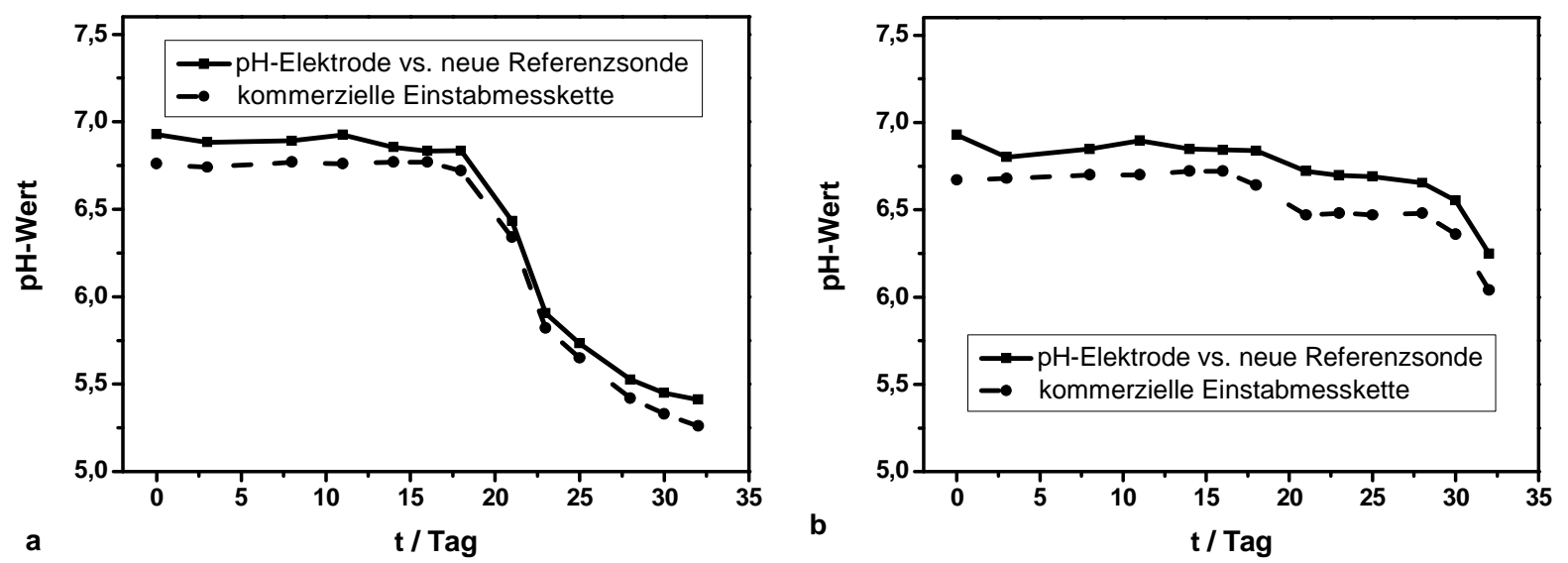

Bild 3 Änderung des pH-Wertes bei der zeitlichen Alterung von verschiedenen Milchproben a) Frischmilch; b) H-Vollmilch

Neben verschiedenen Milchprodukten wurden unter Einsatz der neuen Referenzsonden auch wässrig/alkoholische Gemische mit Alkoholanteilen von $20 \%$ bis $100 \%$ untersucht. Dabei wurden die Elektroden vorwiegend als Referenzelektroden in Kombination mit pH-Glaselektroden als Messelektroden eingesetzt. Es konnte eine Abhängigkeit des Potentialwertes vom Ethanol- bzw. Methanolanteil beobachtet werden, wobei sich die Potentiale bei Erhöhung des Alkoholanteils zu negativeren Werten verschieben. Das konnte auch in vergleichenden Messungen unter Verwendung konventioneller $\mathrm{Ag} / \mathrm{AgCl}-$ Referenzelektroden bestätigt werden. Die mit den neuen Elektroden in organischen Medien ermittelten Potentialwerte sind stabil und reproduzierbar. 
Bild 4 zeigt eine Kalibrierkurve mit an verschiedenen Weinsorten gemessenen pH-Werten. Diese unterscheiden sich nur geringfügig und stimmen mit den mit kommerziellen $\mathrm{pH}$-Einstabmessketten erzielten Werten gut überein.

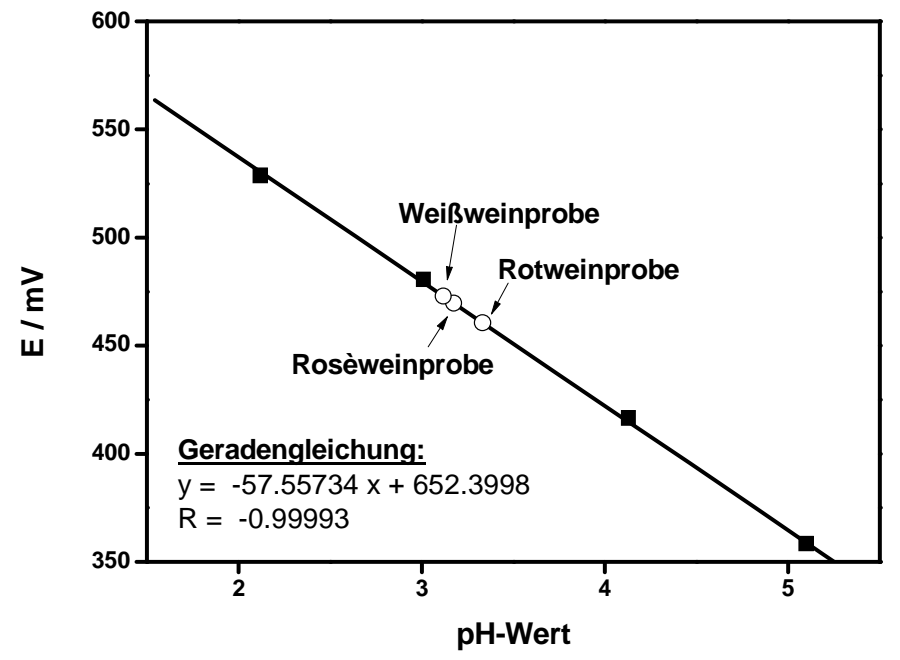

Bild 4 Kalibrierkurve mit an verschiedenen Weinsorten gemessenen pH-Werten, Messung mit einer $\mathrm{pH}$-Glaselektrode und der neuen Referenzsonde auf Basis der Wasserstoffelektrode

\section{Schlussfolgerungen}

Untersuchungen in Lebensmittelproben unter Verwendung der neuen Referenzsonden auf der Basis von Wasserstoffelektroden und von pH-Glaselektroden als Messelektroden zeigten gute Reproduzierbarkeit und hohe Potentialstabilität. Der konstruktive Aufbau der modifizierten WasserstoffReferenzelektroden hat sich beim Einsatz in den verschiedenen wässrigen Lebensmittelproben bewährt. Die mit den neuen Elektroden in organischen Medien ermittelten Potentialwerte sind stabil und reproduzierbar. Damit eröffnen sich gute Perspektiven für zukünftige Applikationen der Referenzsonden auch in nichtwässrigen Lösungen und wässrig/organischen Gemischen. Zukünftiges Ziel ist die Entwicklung einer anwenderfreundlichen symmetrischen Einstabmesskette auf der Basis von Wasserstoffelektroden für Anwendungen in Lebensmittel- und Umweltproben.

\section{Literatur}

[1] M. Le Blanc: Z. Phys. Chem., 12 (1893)

[2] H. Galster: pH-Messung. VCH-Verlag: Weinheim 1990

[3] H. Kaden, W. Vonau: J. prakt. Chem. (1998) 710 - 721

[4] K. Schwabe: pH-Messung. Akademie-Verlag: Berlin 1980

[5] Patent DE 3532335

[6] Patent DE $4112784 \mathrm{C} 1$

[7] Patent US 5.407.555

[8] Patent DE 19724007 C2

\section{Danksagung}

Das dieser Arbeit zugrunde liegende Vorhaben wurde in dankenswerter Weise mit Mitteln des Bundesministeriums für Wirtschaft und Technologie (BMWi) unter dem Förderkennzeichen 2218307ST9 gefördert. Die Verantwortung für den Inhalt dieser Arbeit liegt bei den Autoren. 